\title{
HIGH EMPIRICAL STUDY of STRONG TAQ IDENTIFICATION USING a DISTRIBUTED ANTENNA for RADIO FREQUENCY IDENTIFICATION
}

\author{
Alaa K. Al-Azzawi \\ Department of MechatronicsEngineering Techniques, Technical Engineering College-Baghdad, \\ Middle Technical University, Ministry of Higher Education \& Scientific Research, Baghdad-Iraq. \\ E-mail: ali_ksaleh2010@yahoo.com
}

\begin{abstract}
Radio Frequency Identification (RFID) is a form of wireless communication that desegregates the use of electromagnetic coupling in the radio frequency spectrum to automatically identify and track tags attached to objects. Tags are a type of tracking system that uses smart microchip (bar code data collection) in order to identify items. RFID tags utilize radio frequencies to transmit data from the tag to a reader, which then to an RFID computer program for interpretation. In this paper, we concentrated on the designing of equipment that contains camcode's stainless steel-tags to offer robust performance or exceptional durability in highly incendiary environments. Furthermore, offering excellent resistance to frequent cleaning and strong incendiaries often found in medical, chemicals, and similar environments. This study presents an a distributed antenna structure operating at a frequency of $915 \mathrm{MHz}$ to increase the tag identification sensitivity for passive radio frequency identification (RFID) systems. The tag antennas are formed of balanced lines wrapped around conducting rectangular patches and separated by a gap. The result shows that a high sensitivity RFID tag chip is achievable using a four distributed patch antenna as well as standard CMOS process without optional processes.
\end{abstract}

Keywords: Radio Frequency Identification,distributed antenna systems, gain (G), camcode's stainless steel-tags

\section{INTRODUCTION}

RFID systems elementally consist of four elements: RFID tags, RFID readers, antennas with a proper radio frequency technologies, and RFID computer program for interpretation. RFID appears numerous differences over previous technologies like barcode and camcode's [1]. RFID devices are more accurate and can be read without the need of visual contact [2]. Tags are the main structuring hunk of RFID. Each active tag has two parts; an antenna LC resonant circuit to induce an energizing signal and to send a modulated RF signal,and a micro-silicon device that contains a radio transceiver for sending a response back to the reader, control logic, some amount of memory, and a power system. Furthermore, the tag is activated by a time-varying electromagnetic RF field that is transmitted by the reader. The resonant frequency $\left(f_{o}\right)$ of the LC circuit must be tuned to the carrier frequency of the reader to maximize the magnetic coupling field as well as maximizing the quality factor of the tuned circuit.Most of memories in RFID device is made of complementary metal-oxide semiconductor (CMOS) or ferroelectric random-access memory (FRAM) array.

Typical read/write range is the optimal communication distance between the reader and attached tag. For clarity, reading data out from the tag and writing data from reader to tag is related to; electromagnetic field coupling between the reader and tag antennas, output power from the reader, carrier frequency bands, and power consumption of RFID device.Security and privacy problems of RFID tag search system include them of general RFID system [3], because RFID tag search system is an extension of general RFID system. Recently, the difficulties in designing a secure protocols for RFID tag search werebriefly examined in [4] without help of a central database. Four security protocols that seem to cover almost all the RFID security requirements are studied in this paper:Strong Private Authentication (SPA) [5], Efficient Mutual- Authentication Protocol (EMAP) [6,7], Demetrious's Lightweight Protocol, [8] and Advanced Semi-Randomized Access Control (ASRAC) [9]. The read range in backscatter RFID systems depends on the transmitted power, the frequency used, the gain of the reader and the tag antennae and the sensitivity of the receiver [10,11]. The authors in [12] proposed secure RFID mutual documentation protocol based onsynchronized secret against all possible attacks.

By using a high-gain of antennae, or multiple antennas connected to the reader unit, the reader range can be increased to improve the performance of the RFID system. The performance of a passive RFID system is highly dependent on the antennae. Therefore, choosing a proper antenna for the passive RFID system can increase the reliability of the identification event and improve the efficiency of the manufacturing system. Nowadays, microstrip patch antennas are being pitched for new high speed RFID reader systems [13-14]. 
Authors suggest several improvements to the search protocol to minimize the impact of tracking. One solution is to adopt a challenge-response protocol. They are well congenial for RFID reader systems due to their versatility, low cost and low sensitivity to the manufacturing liberality.

The read range in backscatter RFID systems depends on the transmitted power, the frequency used, the gain of the reader and the tag antennae and the sensitivity of the receiver $[15,16]$. The rest of the paper is organized as follows. In Section 2, we present the proposed RFID system structure. Section 3 shows the top, bottom view and the sizes of the microstrip patch antenna. for testing and implementation, as well as the results of the security and performance analyses. Improvements to the protocols are suggested in Section 4, and conclusions and a proposal for future work are presented in Section 5.

\section{RFID SYSTEM ARCHITECTURE}

Figure 1 shows the proposed distributed antenna structure for a passive RFID system. Four separate patch antennas articulated on the opposite locations were connected to a data collection reader unit through a fourharbor power splitter/combiner, which can amplify the transmitting and receiving signals respectively.

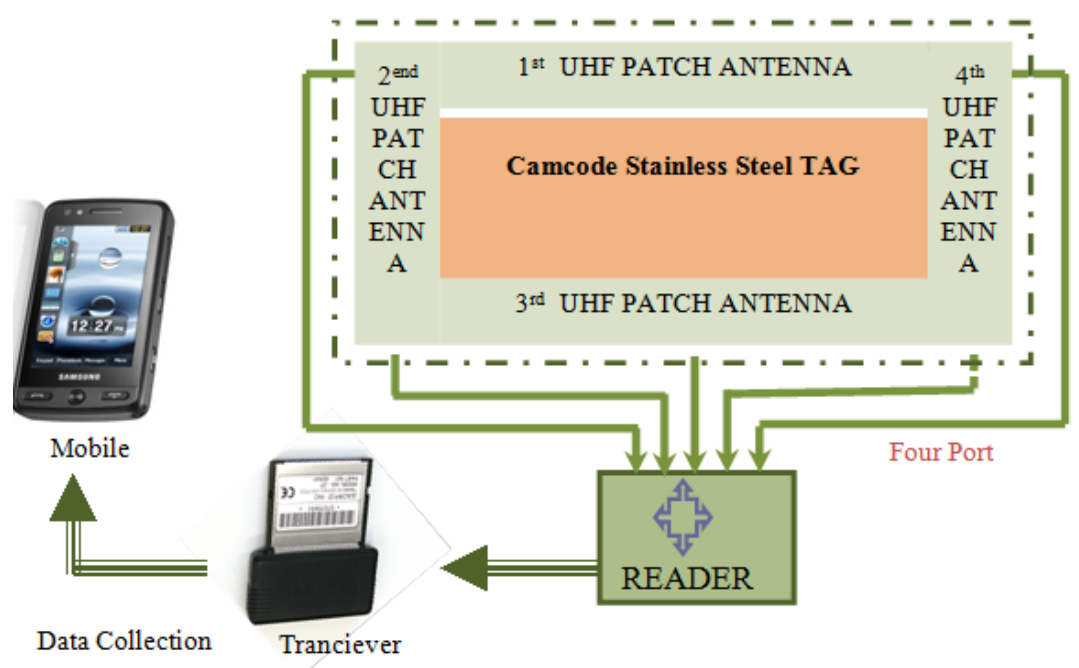

Fig. 1 The proposed RFID system structure

A perfect ultra-high frequency (UHF) RFID system contains readers and passive tags. The latter has two main elements: an antenna of LC resonant circuit to induce an energizing signal and to send a modulated radio frequency(RF) signal, and a micro-silicon device. It also known an application-specific integrated circuit (ASIC). The electronic product code(EPC-Class 1) - Generation2 UHF-RFID standard defines an operational reader-tag distance between 0.5 and $9 \mathrm{~m}$ in an industrial environment. The optimal communication distance (range) between the tag and reader antennas implies that the reader should detect the tag in near and far fields of the reader's antenna. Path loss and fading statistics are two factors that need to be addressed.The key challenge in RFID systems is that the range of most current passive UHF-RFID systems (maximum distance to read data out from the tag and maximum distance to write data from reader to tag) is related to electromagnetic field coupling between the reader and tag antennas as well as limited to a large extent by the path loss from the reader to the tag.

An antenna uses radio transmissions at a working frequency of $915 \mathrm{MHz}$ to transmit energy to tags, afterwardsemitting a nonpareil identification code back to a data collection reader unit. Thus the reader is connected via a mobile for data processing through a standard interface of universal serial bus(USB). Moreover, the four-harbor power splitter/combiner consists of Wilkinson power splitters (WPS), band pass filters (BPF) and power amplifiers (PA). The WPS is provided with theperformances of better impedance matching of three harbors $(50 \Omega)$, lower power loss $(<3 d B)$ and superlatively isolation $(>35 d B)$. It therefore connected to thebidirectional amplifiers due to therestitution of splitter and BPF losses. The bidirectional amplifier must has a high power output and low nonlinear distortion.

The maximum magnetic flux density $(\varnothing)$ that is passing through the tag coil is acquired when both reader and tag coils are reposed in parallel with respect to each other. This proviso results in maximum induced voltage in the tag coil and also maximum read range. The inner product expression via equation 1 can be expressed in terms of a mutual electromagnetic coupling field between the reader and tag coils. The mutual coupling between the two coils is maximized in the above proviso. 


$$
\Psi=\int B \cdot d S
$$

Where, $B$ is the magnetic field, $S$ is the surface area of the coil, and $(\cdot)$ is the inner product (cosine angle between two vectors) of vectors $B$ and surface area $S$.

\section{PATCH ANTENNA}

The mutual inductance is the inductance that is yielded from the magnetic fields produced by adjoined conductors. The mutual inductance is robust orpositive when the directions of current on conductors are in the same direction, and weak or negative when the directions of currents are opposite directions. The mutual inductance between two parallel conductors is a function of the length of the conductors and of the geometric mean distance between them. The mutual inductance of two conductors is calculated via equation 2 .

$$
M=2 l * M_{P} \ldots \ldots \ldots \ldots \ldots F
$$

where $l$ is the length of conductor in centimeter. $M_{P}$ is the mutual inductance parameter and calculated via3

$$
M_{P}=\ln \left\{\left(\frac{l}{G_{D}}\right)+\left[1+\left(\frac{l}{G_{D}}\right)^{2}\right]^{1 / 2}\right\}-\left[1+\left(\frac{l}{G_{D}}\right)^{2}\right]^{1 / 2}+\left(\frac{G_{D}}{l}\right)
$$

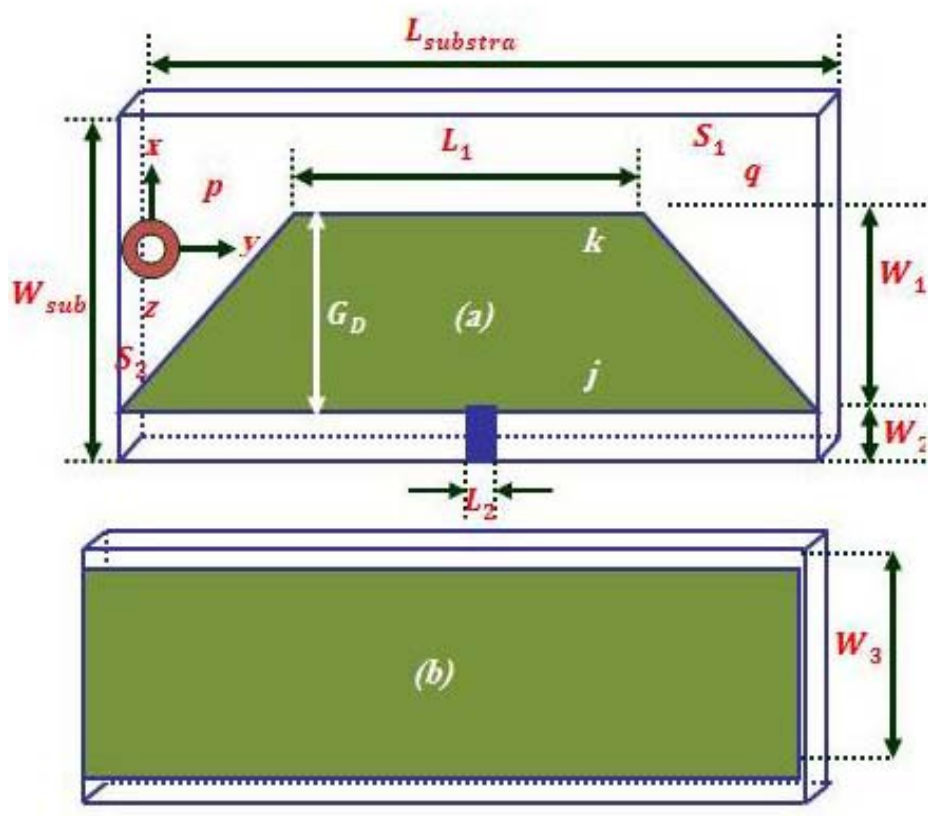

Fig. 2 The proposed microstrip patch antenna with a working frequency at915 $\mathrm{MHz}$ (a) Top view. (b) Bottom view

where $G_{D}$ is the geometric mean distance between two conductors, which is approximately equal to the distance between the track center of the conductors. Let us consider the two conductor segments shown in Figure 2.

$j$ and $k$ in the above figure are indices of conductor, $p$ and $q$ are the indices of the length for the difference in the length of the two conductors. The above configuration (with partial segments) occurs between conductors in multiple turn helixes inductor. The mutual inductanceof conductors $j$ and $k$ in the above configuration is:

$$
M_{j, k}=\frac{1}{2}\left\{\left(M_{k+p}+M_{k+q}\right)-\left(M_{p}+M_{q}\right)\right\}
$$

If the length of $l_{1}$ and $l_{2}$ are the same $\left(l_{1}=l_{2}\right)$, then Equation 4 abbreviates to:

$$
M_{j, k}=M_{k} \quad \text { for } \quad p=q=0
$$

The basic design considerations for transmit-receive antenna are delineated using a commercial high frequency simulation software(HFSS). In this study, we chose to use a microstrip patch antenna with atrapezoid shape because it is easiest to implementation, easiest to mounting on any surface and increased antenna bandwidth (BW) of lower than $210 \mathrm{~dB}$ level, and thus achieved the exact working frequency of around 
$918 \mathrm{MHz}$. With a proceeding ground plane, microstrip patch antennas can be designed to operate in the vicinity of metal. Here, the size of a patch antenna depends upon both the wavelength used and dielectric characteristics of the antenna substrate. Fig. 2shows the geometry of the proposed patch antenna. The chosen antennas were made of substrate FR-4 with a thickness of $1.58 \mathrm{~mm}$ and a relative permittivity $\left(\varepsilon_{r}\right)$ of 4.368 . They were designed to operate in a range between $(915-918 \mathrm{MHz})$. The total volume of this antenna is $\left(96 \times 42 \times 1.58 \mathrm{~mm}^{3}\right)$ and it is printed on both the top face (the radiating element and feeding line) and bottom face (the ground plane). The values of each physical dimension of the proposed antenna are shown in Table 1.

TABLE 1. Sizes of the proposed patch antenna

\begin{tabular}{|c|c|}
\hline Item & Size \\
\hline FR -4 Thickness & $1.58 \mathrm{~mm}$ \\
\hline$W_{\text {sub }}$ & $42 \mathrm{~mm}$ \\
\hline$L_{1}$ & $54 \mathrm{~mm}$ \\
\hline$L_{2}$ & $1.24 \mathrm{~mm}$ \\
\hline$W_{1}$ & $22 \mathrm{~mm}$ \\
\hline$W_{2}$ & $4.8 \mathrm{~mm}$ \\
\hline$W_{3}$ & $32 \mathrm{~mm}$ \\
\hline$L_{\text {sub }}$ & $96 \mathrm{~mm}$ \\
\hline
\end{tabular}

\section{1 The Design Specifications of Micro-strip Antenna}

Micro-strip antennas are conformable, mechanically robust, and very versatile. They are useful for high performance in extreme applications such as electronic devices, aircraft, missiles, cellphones [17][18], Wi-Fi [19], Bluetooth [20] and many other applications. Dimensions of a micro strip patch antenna depend on the resonant frequency and value of the dielectric medium called a substrate. The basic considerations of our design for a rectangular micro strip patch antenna (RMPA) were waylays: Firstly, in using a full-wave model. This model is the most accurate, versatile, easiest to simulate, and also the most complex and has less physical insight. Secondly, evaluation of the most benefits parameters in order to improve the bandwidth hopefully, to carrying out and proposed several techniques. The following procedure assumes we know the dielectric constant $\left(\epsilon_{r}\right)$, the resonant frequency $\left(f_{o}\right)$, and the height of the substrate $(h)$. Further, the procedure includes theimplementation of thick substrate with a low dielectric constant.Figure 3 shows the cues to how to calculate the parameters of a micro strip patch antenna via the following equations 6, 7, 8, 9 and 10 .

\subsection{Calculation of width $(W)$}

The width of the micro strip patch antenna $(\boldsymbol{W})$ is calculated via equation 6 [20][21][22].

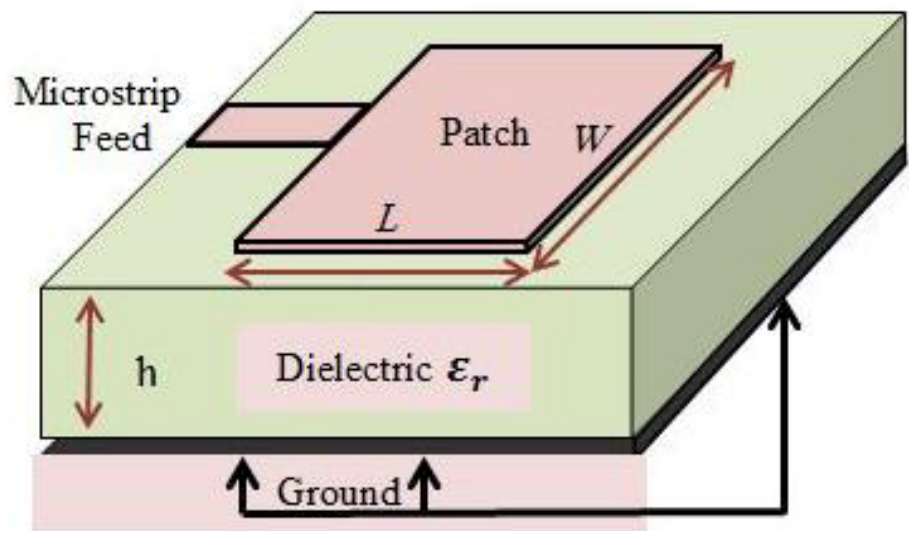

Fig. 3 Micro strip patches antenna calculation 


$$
W=\frac{1}{2 f_{o} \sqrt{\mu_{o} \epsilon_{o}}} \sqrt{\frac{2}{\epsilon_{r}+1}}=\frac{C}{2 f_{o}} \sqrt{\frac{2}{\epsilon_{r}+1}}(6)
$$

Where, $\mathbf{f}_{\mathbf{o}}$ is the resonance frequency, $\mathbf{C}$ is the free space velocity of light $\left(\mathbf{3} \times \mathbf{1 0 ^ { 8 }} \mathbf{m l s e c}\right)$, and $\varepsilon_{\mathrm{r}}$ is the relative permittivity of the dielectric medium of substrate.

\subsection{The effective dielectric constant $\left(\varepsilon_{\text {eff }}\right)$}

The calculation of the effective dielectric constant $\left(\boldsymbol{\varepsilon}_{\boldsymbol{e f f}}\right)$ of a rectangular micro-strip antenna is based on both the height, and the calculation of the width for the patch antenna. This patch is selected to be very thin such that $\left(T h<<\lambda_{o}\right)$ where, Th is the patch thickness. The height $h$ of the dielectric substrate is given in a range $\left(0.003 \lambda_{o} \leq h \leq 0.05 \lambda_{o}\right)$. While the dielectric constant is usually in the range $\left(2.2 \leq \varepsilon_{r} \leq 12\right)$. The evaluation is implemented via equation 7 .

$$
\varepsilon_{e f f}=\frac{\varepsilon_{r}+1}{2}+\frac{\varepsilon_{r}-1}{2}\left[1+12 \frac{T h}{W}\right]^{-\frac{1}{2}}(7)
$$

where, $T h$ is the thickness and $\boldsymbol{W} / \boldsymbol{T} \boldsymbol{h}>1$.

\subsection{The calculation of the length extension (electrical distance) $\Delta L$ is calculated via 8}

$$
\frac{\Delta L}{T h}=0.412 \frac{\left(\varepsilon_{\text {eff }+0.3}\right)\left(\frac{W}{T h}+\mathbf{0 . 2 6 4}\right)}{\left(\varepsilon_{\text {eff }-0.258}\right)\left(\frac{W}{T h}+\mathbf{0 . 8}\right)}(8)
$$

Here, the electrical distance $(\Delta L)$ is a function of the effective dielectric constant $\left(\boldsymbol{\varepsilon}_{\boldsymbol{r} \boldsymbol{e} f \boldsymbol{f}}\right)$ and width-to-height (thickness) $\frac{W}{T h}$.

3.5The calculation of actual length $L$ of the patch is calculated via 9

$$
L=\frac{1}{2 f_{o} \sqrt{\epsilon_{\text {reff* }}} \sqrt{\mu_{o} \epsilon_{o}}}-2 \Delta L(9)
$$

The approximate lengths of the microstrip $L$ vary between $(0.47-0.49) \frac{\lambda_{0}}{\sqrt{\varepsilon_{r}}}$.

\subsection{The calculation of the effective length of the patch is via 10.}

$$
L_{e f f}=\frac{c}{2 f_{o} \sqrt{\varepsilon_{\text {reff }}}}(10)
$$

where, $\boldsymbol{L}_{\boldsymbol{e f f}}$ is the effective length of the patch?

3.7 The calculation of the ground length and width are via equations 11 and 12 respectively.

$$
\begin{aligned}
& G_{L}=6 h+L(11) \\
& G_{W}=6 h+W
\end{aligned}
$$

Referring to the above equations, the dimensions of our proposed microstrip antenna design are: $L=$ $0.0244 \mathrm{~mm}$ and $W=0.1288 \mathrm{~mm}$, substrate with height $h=0.00523 \mathrm{~mm}$ and effective dielectric constant $\epsilon_{\text {reff }}=4.99546$, is operating at $915 \mathrm{GHz}$ frequency. The antenna described in this paper is designed for optimum performance on the surface of any object, regardless of whether it is conductive or has high dielectric constant or loss.The RFID ASIC used is a passive UHF read only transponder (EM4222 from EM Microelectronics [16]) with the parameters given in Table 2. In this study, it is possible to design a dual frequency tag by matching at the center frequency between bands. All tags were simulated using CST Microwave Studio and input match was optimized using the quasi-Newton optimizer embedded into the application. After optimization by simulation, designs were fabricated for practical validation. RFID ASIC input impedance varies as a function of input power and in practice some physical tuning was required to provide optimum experimental read range.

Table 2. EM4222 data

\begin{tabular}{|l|l|l|l|}
\hline ID - Number & Data rate & Frequency & i/p impedance \\
\hline 64 bit & 256 kbit $S^{-1}$ & $869 \mathrm{MHz}$ & $128-j 577 \Omega$ \\
\hline 64 bit & 256 kbit $S^{-1}$ & $915 \mathrm{MHz}$ & $132-j 577 \Omega$ \\
\hline
\end{tabular}


Referring to Fig. 2 the width of slots 1 and 2 depend on the chosen substrate type and thickness and are altered by simulation to obtain the required match. Further, The input impedance of RFID ASICs varies with device and is a function of received reader power. It was therefore necessary in practice to carry out some systematic physical tuning. This was done by fabricating the simulated design and trimming the feed line width $W_{2}$ (Fig. 2) to maximize read range.

\section{MATERIALS AND METHODS}

Over the past few years, the sophistication of RFID technology has been developed very fast, adding new features to conventional automatic identification and data captivating applications. The reader and the tags were developed to buttressing the modified tag collection algorithm that was applied with the slot mechanism and broadcast sleep mechanism, as well as the standard tag collection algorithm.

RF transceiver controller eliminates the load on the microprocessor in the reader by processing RF communication transactions separately to help readers with embedded operating systems, so the reader's microprocessor can use its processing resources for other tasks running on the operating system.

RFID tags vary widely by design, and so three types of passive RFID tags with different types of antennae and chips were examined in this study, all configured to fit standards forEPC Class 1 Gen 2 [21].

EPC Class 1 Gen 2 tags have four memory banks: reserved, EPC, TID and user memory. The EPC bank, typically 96 bit in size, is the one that mainly characterizes EPC Gen 2 tags [22]. The tag shown in Fig. 4 is the standard EPC Gen2 UHF RFID (V750-D22M01-IM) is used and perfectly matched to the chip at $915 \mathrm{MHz}$

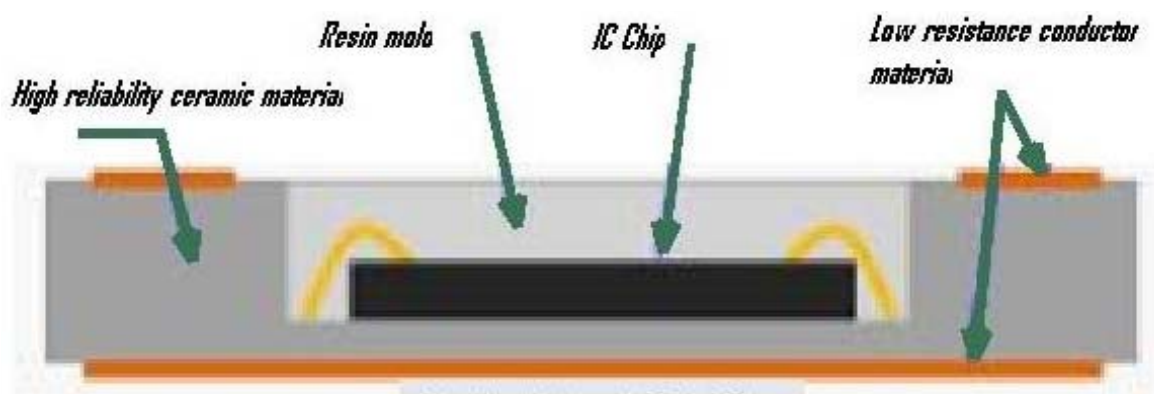

(a)

Utor-Small Ceramic UHF AFID Tag

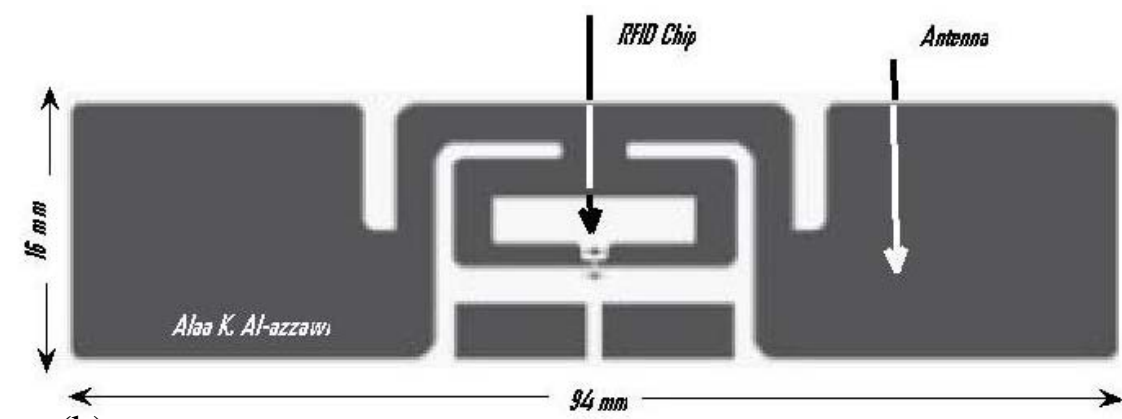

(b)

Fig. 4 UHF RFID tag (a) Ultra small ceramic, (b) Printed antenna[23].

In summary, choosing the appropriate RFID security protocol is based on different factors: the application of the RFID system (i.e., commercial, security or tracking), and on the complexity fetters imposed on the tag. Based on these two factors, one can decide which security features are important and what are the security exemplars that can be used in the tag, such as cryptographic hashes or simple logic functions. Camcode's stainless steel tags are the ideal solution for applications in chemical, food processing environments, or those applications requiring resistance to frequent cleaning with strong caustics. These tags were constructed of ceramic-like material that is thermally fused to a high quality stainless steel plate with standard thickness of (0.018"). Furthermore, high performance indelible pressure-sensitive adhesive as well as mechanical connectedly were available. Here, camcode's stainless steel tags will remain readable after subjected in crueler environments and intermittent heat up to $\left(700^{\circ} \mathrm{F}\right)$. 


\section{MEASUREMENT RESULTS}

The resonance frequency and the bandwidth of the antenna are given by the parameters ' $f$, ' and ' $B$ ', respectively.

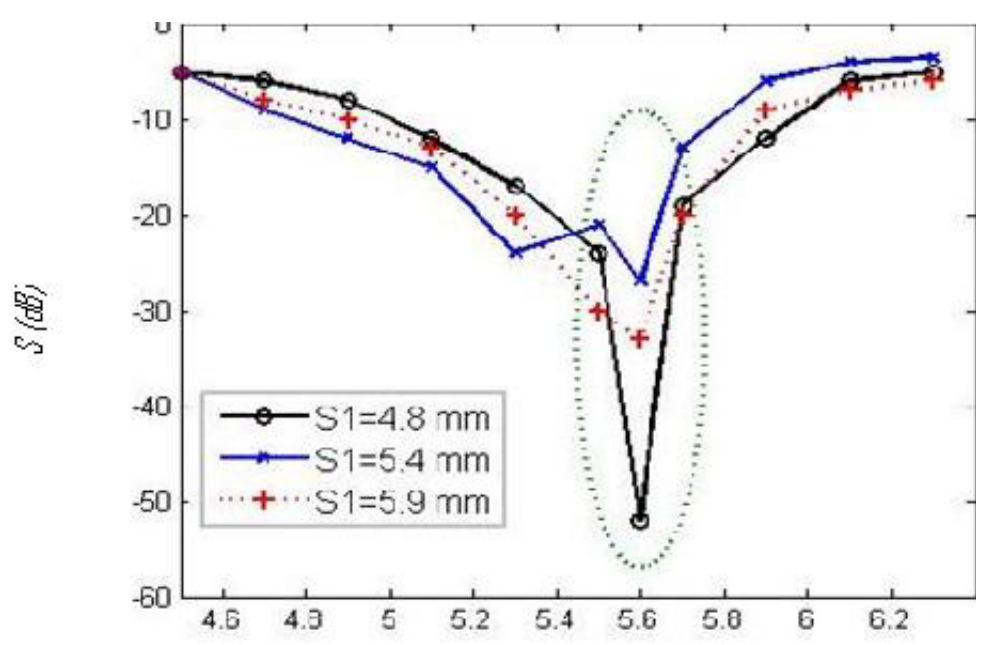

Frequency (OHz,

Fig. 5 The effect of changing the parameter ' $S_{1}$ '

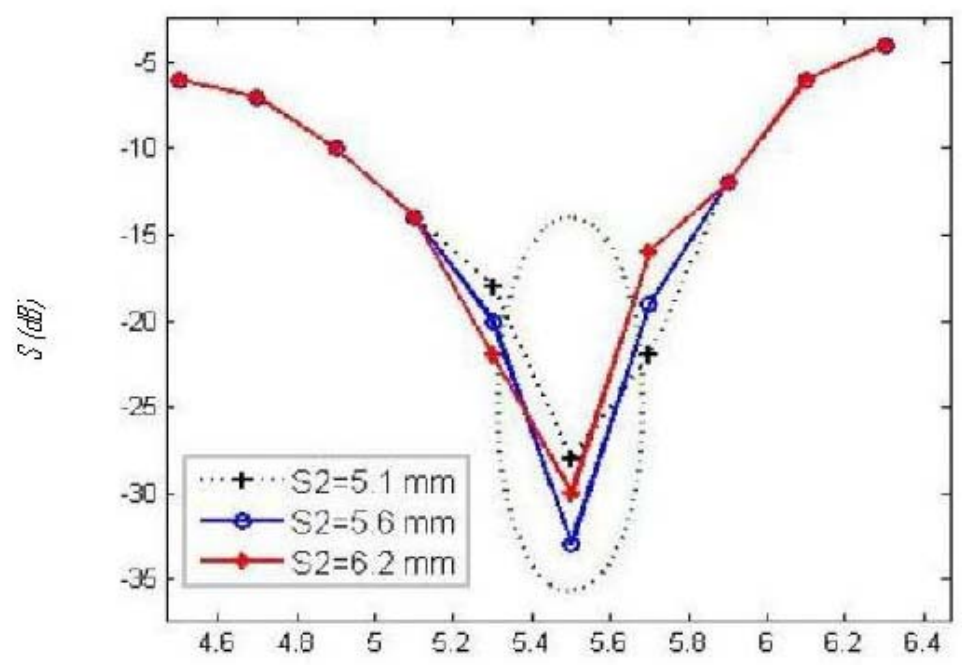

Fraquence $(0 \mathrm{hz}$,

Fig. 6 The effect of changing the parameter ' $S_{2}$,

As the parameter ' $f_{\circ}$ ' increases, the operating band shifts to a lower frequency range; meanwhile the parameter ' $B$ ' controls the bandwidth and the level of return loss at the main resonance frequency. Modifying the gap width ' $G_{W}$ ' and the dimensions of the dual structure ' $S_{1}$ ' and ' $S_{2}$ ', one can tune the antenna for a better input impedance to match or vary the frequency. Increasing ' $G_{W}$ ' shifts the resonating frequency to an upper frequency together with a slight reduction in the return loss value. The double structure parameters ' $S_{1}$ ' and ' $S_{2}$ ' are opposite in behavior, so a trade-off is adopted. Increasing the value of ' $S_{1}$ ' results in a reduction of both the frequency of operation as well as the impedance matching. The following Figures 5, 6, and 7 show the parametric study of ' $S_{1}$ ', ' $S_{2}$ ', and ' $G_{W}$ '. 


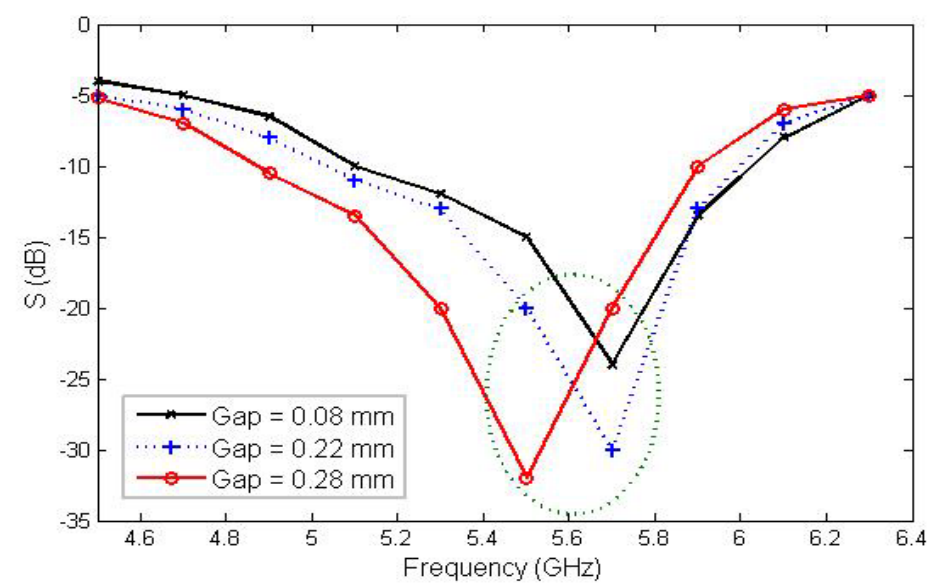

Fig. 7 The effect of changing the parameter ' $G_{W}$ '

The simulated curves in Fig. 8 shows that small slot size S2, is important in matching the tag to the ASIC although it does not significantly affect resonant frequency.

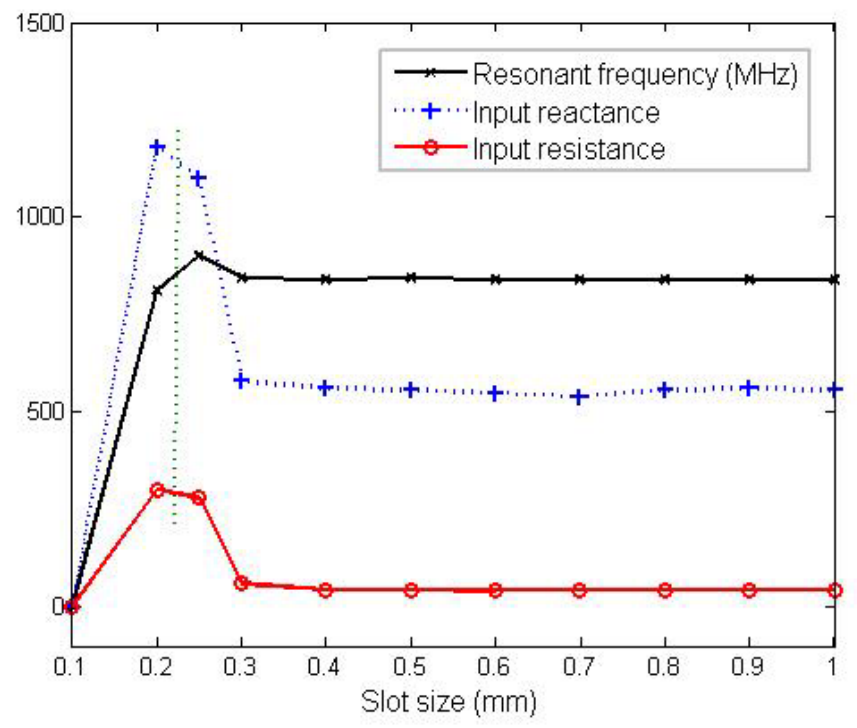

Fig 8 Simulated effect of slot size S2 on antenna impedance andresonant frequency, solid black line is the resonant frequency $(\mathrm{MHz})$, dotted blue line is the input resistance $(\Omega)$, solid red line is the input reactance $(\Omega)$.

\section{CONCLUSION}

In this paper a rectangular microstrip patch antenna is successfully designed at a resonant frequency of 915 GHz. The presented antenna analysis shows a sanitary gain of $12.056 \mathrm{~dB}$ with minimal harmonic suppression. The voltage standing wave ratio (VSWR) of the antenna is in the range between (1.08-1.26). Discussion has been focused on performance ofcamcode's stainless steel tags to seek the best topology suit for wireless systems. However, some modification has been made to construct harmonic rejection capability in antenna design. Improvement in overall antenna design that need to be considered including low conversion efficiency due to excitation at harmonic frequency and polarization mismatch. The simulated and measurement result in Figs. 5, 6,7 , and 8 show good performance of antenna design as well as achieves harmonic rejection efficiency.Finally, the cue of a distributed antenna structure for a passive RFID is proposed. The cue presents a four-harbor power combiner/splitter with power reimbursing amplifiers between the reader chip and distributed patch antennas. Further, the overall design of RFID tag antenna was printed on a thin flexible polyester. 


\section{ACKNOWLEDGMENTS}

I gratefully acknowledge the support of all the staff of the department of electronics and communication engineering for their support and contribution through my research period. Also I would like to express my thanks to the reviewers of the journal for their all-out efforts and all-out precise reading to support us for completing the research article, and their robust suggestions for the improvement of our work.

\section{REFERENCES}

[1] Luis R. G., Loredana L., "The role of RFID in agriculture: Applications, limitations and challenges", Computers and Electronics in Agriculture, vol. 79, pp. 42-50, 2011.

[2] Abad E., et al., "RFID smart tag for traceability and cold chain monitoring of foods", Journal of Food Engineering, vol. 93, no. 4, pp. 394-399, 2009.

[3] A. Juels. RFID security and privacy: A research survey. IEEE Journal on Selected Areas in Communication, 24(2), February 2006.

[4] Chiu C. Tan, Bo Sheng, and Qun Li. Secure and Serverless RFID Authentication and Search Protocols. IEEE Transactions on Wireless Communication, Vol. 7, No 4, APRIL 2008.

[5] Li Lu, Jinsong Han, Lei Hu, Yunhao Liu, and Lionel M; “Dynamic Key-Updating: Privacy-Preserving Authentication for RFID Systems"; March 2007.

[6] J. Yang, J. Park, H. Lee, K. Ren, and K. Kim, "Mutual authentication protocol for low-cost RFID”, Ecrypt Workshop on RFID and Lightweight Crypto, 2005.

[7] Peris-Lopez, P., Hernandez-Castro, J.C., Estevez- Tapiador, J.M., Ribagorda, A.:”EMAP: An efficient mutual authentication protocol for low-cost RFID tags”. In: OTM Federated Conferences and Workshop: IS Workshop - IS’06. Vol. 4277 of LNCS, (2006) 352-361.

[8] Dimitriou, T.. “A Secure and Efficient RFID Protocol that could make Big Brother (partially) Obsolete”. In Int.Conf. on Pervasive Comp. and Comm., 2006, March 2006. IEEE, IEEE Computer Society Press.

[9] Yong Ki Lee, Ingrid Verbauwhede, "Secure and Low cost RFID Authentication Protocols"; University of California, Los Angeles; 2005.

[10] D. I. Kim, S. W. Jung, and Y. Yun, “A high performance transformer-type Wilkinson power splitter with compensating circuit for CATV transmission system,” IEEE trans. on consumer electronics, vol. 50, no. 3, pp. 934-940, Aug. 2004.

[11] K.V. S. Rao, P. V. Nikitin, and S. F. Lam, “Antenna design for UHF RFID tags: a review and a practical application,” IEEE trans. on Antennas and Propagation, vol. 53, pp. 3870-3876, Dec. 2005.

[12] S. Lee, T. Asano, K. Kim, "RFID Mutual Authentication Scheme based on Synchronized Secret Information", In Symposium on Cryptography and Information Security, Hiroshima, Japan, January (2006).

[13] BUDAK E., CATAY B., TEKIN I., ET AL.: 'Microstrip patch antenna for RFID applications'. Proc. RFID Eurasia Conf., September 2007, pp. 1-3

[14] SHARAWI M.S.: 'Use of low-cost patch antennas in modern wireless technology', IEEE Potentials, 2006, 25, (4), pp. 35-47

[15] NILSSON H.-E., SIDEN J., OLSSON T., JONSSON P., KOPTIOUG A.: 'Evaluation of a printed patch antenna for robust microwave RFID tags', IET Microw. Antennas Propag., 2007, 1, (3), pp. 776-781

[16] PENTTILA“ K., KEASKILAMM M., SYDA“NHEIMO L., KIVIKOSJI M.: 'Radar cross-section analysis for passive RFID systems', IEE Proc. Microw. Antennas Propag., 2006, 153, (1), pp. 103-109

[17] S.-C. Jung, M.-S. Kim, and Y. Yang, "Baseband noise reduction method using captured TX signal for UHF RFID reader applications," IEEE Transactions on Industrial Electronics, vol. 59, no. 1, pp. 592 -598, 2012.

[18] Ramna, Amandeep Singh Sappal "DESIGN of RECTANGULAR MICROSTRIP PATCH ANTENNA USING PARTICLE SWARM OPTIMIZATION ”International Journal of Advanced Research in Computer and Communication EngineeringVol. 2, Issue 7, July, 2013.

[19] JaswinderKaur, Rajesh Khanna “Co-axial Fed Rectangular Microstrip Patch Antenna for 5.2 GHz WLAN Application “ Universal Journal of Electrical and Electronic Engineering 1(3):94-98, 2013 DOI: 10.13189/ujeee.2013.010306 http://www.hrpub.org

[20] Md. MarufAhamed, Kishore Bhowmik, Abdulla Al Suman "Analysis And Design of Rectangular Microstrip Patch Antenna On Different Resonant Frequencies For Pervasive Wireless Communication” INTERNATIONAL JOURNAL OF SCIENTIFIC \& TECHNOLOGY RESEARCH VOLUME 1, ISSUE 5, JUNE 2012.

[21] NKITIN P.V., RAO K.V.S.: 'Performance limitations of passive UHF RFID systems'. Proc. IEEE Antennas and Propag. Society Symp., July 2006, pp. 1011-1014.

[22] http://www.emmicroelectronic.com/, accessed March 2009.

[23] EPCglobal Inc., EPC Radio-Frequency Identity Protocols: Class-1 Generation-2 UHF RFID Protocol for Communications at 860 MHz-960 MHz Version 1.2.0, October 2008. [Online]. Available: http://www.epcglobalinc.org/standards/uhfc1g2

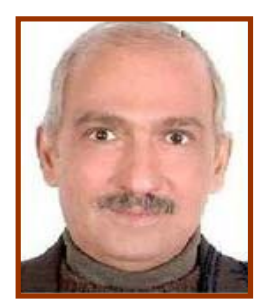

\section{ABOUT THE AUTHOR}

ALAA K. AL-AZZAWI (Member, IEICE) received the B.Sc. and M.Sc. degrees (honors) in Communications Engineering department from the University of Engineering Technology, Baghdad-Iraq, in 1979, 2002, respectively, and the Ph.D. degree in wireless communications engineering, from the University Putra Malaysia (UPM), in 2012. From 1980 to 2008, he was a lecturer at the department of Electrical and Electronic Engineering, Ministry of Higher Education \& Scientific Research, Currently he was assistant Prof. at the Mechatronics Engineering department. His current research interests are in Digital Image Processing, Medical Imaging, Artificial intelligent, neural network applications, engineering and information technologies, advanced Signal Processing, WLAN Net., and TelCommunications. 